\title{
Short-Term Treatment with Esmolol Reverses Left Ventricular Hypertrophy in Adult Spontaneously Hypertensive Rats via Inhibition of Akt/NF- $\kappa$ B and NFATc4
}

\author{
Begoña Quintana-Villamandos $\mathbb{D}^{1,2}$ David A. Goukassian, ${ }^{3}$ \\ Sharath P. Sasi, ${ }^{4}$ and Emilio Delgado-Baeza ${ }^{5}$ \\ ${ }^{1}$ Departamento de Anestesiología, Hospital General Universitario Gregorio Marañón, Doctor Esquero 46, 28007 Madrid, Spain \\ ${ }^{2}$ Departamento de Farmacología, Facultad de Medicina, Universidad Complutense de Madrid, Madrid, Spain \\ ${ }^{3}$ Center for Translational Medicine, Temple University School of Medicine, Philadelphia, PA 19140, USA \\ ${ }^{4}$ Pfizer, CVMED, 610 Main Street, Cambridge, MA, USA \\ ${ }^{5}$ Instituto de Investigación Sanitaria Gregorio Marañón, Madrid, Spain
}

Correspondence should be addressed to Begoña Quintana-Villamandos; begoquinti@gmail.com

Received 28 October 2017; Revised 24 December 2017; Accepted 3 January 2018; Published 18 February 2018

Academic Editor: François Roubille

Copyright (C) 2018 Begoña Quintana-Villamandos et al. This is an open access article distributed under the Creative Commons Attribution License, which permits unrestricted use, distribution, and reproduction in any medium, provided the original work is properly cited.

\begin{abstract}
Our group has previously demonstrated that short-term treatment with esmolol reduces left ventricular hypertrophy (LVH) in spontaneously hypertensive rats (SHRs). The present study aimed to assess the molecular mechanisms related to this effect. Fourteen-month-old male $\mathrm{SHR}_{\mathrm{s}}$ were treated intravenously with saline as vehicle (SHR) or esmolol (SHR-E) ( $\left.300 \mu \mathrm{g} / \mathrm{kg} / \mathrm{min}\right)$. Agematched vehicle-treated male Wistar-Kyoto (WKY) rats served as controls. After 48 hours of treatment, the hearts were harvested and left ventricular tissue was separated and processed for Western blot analysis to determine the levels of Akt, NF- $\kappa \mathrm{B}, \mathrm{NFATc4}$, Creb1, Serca2a, Erk1/2, and Sapk/Jnk. Biomarkers of oxidative stress, such as catalase, protein carbonyls, total thiols, and total antioxidant capacity were evaluated. Esmolol reversed the levels of p-NFATc4, p-Akt, and p-NF- $\kappa$ B in SHRs to the phospholevels of these proteins in WKY rats without modifying p-Erk1/2, p-Sapk/Jnk, p-Creb1, or Serca2a in SHR. Compared with SHR, esmolol increased catalase activity and reduced protein carbonyls without modifying total thiols or total antioxidant capacity. Short-term treatment with esmolol reverses LVH in aged SHRs by downregulation of Akt/NF- $\kappa$ B and NFATc4 activity. Esmolol treatment also increases catalase activity and reduces oxidative stress in SHRs with LVH.
\end{abstract}

\section{Introduction}

Left ventricular hypertrophy (LVH) is a compensatory response to chronic arterial hypertension. It is an independent risk factor for cardiovascular morbidity and mortality including heart failure, coronary artery diseases, stroke, arrhythmias, and sudden cardiac death [1]. Decrease of $\mathrm{LVH}$ is associated with lower cardiovascular risk; therefore, therapies directed towards decreasing $\mathrm{LVH}$ are accepted as an essential treatment goal in this condition [2]. Decreases in LVH with antihypertensive therapy (angiotensin-converting enzyme inhibitors, angiotensin receptor blockers, $\beta$-adrenergic blockers, calcium channel blockers, and diuretics) have been reported, albeit after long-term treatment $[3,4]$. Our group reported the first study to show regression of LVH after a markedly short period of treatment with esmolol [5].

Esmolol is an ultrashort-acting cardioselective $\beta$-adrenergic blocker with a half-life of approximately 2 minutes, a time to peak effect of about 6-10 minutes, and a washout time of 9 minutes [6]. The pharmacodynamic and pharmacokinetic profiles of this drug make it a suitable cardioprotective agent in clinical practice $[7,8]$.

Our group previously demonstrated that short-term treatment (48 hours) with esmolol reduces LVH in spontaneously hypertensive rats (SHR) [5]. However, the molecular mechanism(s) of this effect have not been elucidated to date. 
Asymmetrical dimethylarginine (ADMA) is an independent risk factor for the development of LVH. Dimethylarginine dimethyl aminohydrolase (DDAH) plays an important role in the pathogenesis of hypertensive LVH by modulating tissue levels of ADMA [9]. We have previously reported that esmolol reduces ADMA concentrations and increases DDAH concentrations in the left ventricle in SHR model [10, 11]. However, the molecular mechanisms of esmolol-mediated regression of LVH remain unknown.

It is increasingly evident that redox-dependent modifications in cellular proteins and signaling pathways play an important role in cardiac hypertrophy [12]. This can have a significant negative impact on cardiac gene expression that can affect various cellular processes involved in cardiac hypertrophy signaling. A number of transcription factors (e.g., nuclear factor of activated T cells 4 [NFATc4], nuclear factor kappa B $[\mathrm{NF}-\kappa \mathrm{B}]$, and mitogen-activated protein kinases [MAPKs, e.g., Akt and Erk1/2]) are involved in cardiac hypertrophy signaling [13-15]. The effect of esmolol on the regulation of these transcription factors and MAPKs in LVH signaling remains unknown.

In this study we tested the hypothesis that esmolol could lead to early regression of $\mathrm{LVH}$, at least in part, through MAPK-mediated regulation of NFATc4 and NF-kB transcription factors. Therefore, the present study aimed to assess whether short-term treatment with esmolol can reverse left ventricular hypertrophy in aged SHR hearts and whether these effects are mediated through regulation of Akt/NF- $\kappa \mathrm{B}$ and NFATc4.

\section{Methods}

2.1. Experimental Animals and Treatment. The study animals: 14-month-old male spontaneously hypertensive rats (SHR) $(n=12)$ and normotensive Wistar-Kyoto (WKY) control rats $(n=6)$ were bred at the animal facility of Universidad Autonoma de Madrid. All rats were supplied with standard rat chow and drinking water ad libitum and were maintained on a $12 \mathrm{~h} / 12 \mathrm{~h}$ light/dark cycle. The animals were housed at a constant temperature of $24^{\circ} \mathrm{C}$ and relative humidity of $40 \%$. Anesthesia was administered by intraperitoneal injection of diazepam $10 \mathrm{mg} / \mathrm{kg}$ (Valium $10 \mathrm{mg} / \mathrm{ml}$; Roche Pharmaceuticals, Madrid, Spain) and ketamine $80 \mathrm{mg} / \mathrm{kg}$ (Ketolar $50 \mathrm{mg} / \mathrm{ml}$; Parke-Davis, Madrid, Spain), and a catheter was inserted into the right internal jugular vein. SHRs were divided into 2 groups: rats treated with esmolol (SHR-E) and hypertensive control rat group (SHR) treated with saline solution (saline as vehicle). SHR-E received an intravenous infusion of esmolol at $300 \mu \mathrm{g} / \mathrm{kg} / \mathrm{min}$ (Breviblock $10 \mathrm{mg} / \mathrm{ml}$; Baxter, Belgium) for 48 hours, and control SHR and WKY received saline solution (vehicle). After 48 hours of treatment, rats were euthanized by decapitation after sedation with an intraperitoneal injection of diazepam $10 \mathrm{mg} / \mathrm{kg}$ and ketamine $80 \mathrm{mg} / \mathrm{kg}$. The hearts were harvested within minutes; left ventricles were bisected and processed for Western blot analysis and oxidative stress biomarkers. All procedures fulfilled the stipulations of the Guide for the Care and Use of Laboratory Animals (Directive 2010/63/EU and RD 53/2013) and were approved by the Ethics Committee of Hospital General Universitario Gregorio Marañón, Madrid, Spain.

2.2. Systolic Arterial Pressure and Heart Rate Measurements. Systolic arterial pressure and heart rate were measured using the tail-cuff method with a photoelectric sensor (Niprem 546, Cibertec, Madrid, Spain). Several determinations were made at each session before and after treatment $(0,12,24,36$ and $48 \mathrm{~h}$ ), and the findings were considered valid if 10 consecutive measurements were within $10 \mathrm{mmHg}$ of each other.

2.3. Sample Preparation. Left ventricular tissue samples were homogenized using a TissueLyser LT system (QIAGEN, Hilder, Germany) programmed with $50 \mathrm{~s}^{-1}$ oscillation for $4 \mathrm{~min}$ in a lysis buffer containing $20 \mathrm{mM}$ Tris- $\mathrm{HCl}$ buffer $(\mathrm{pH}$ 7.5), $150 \mathrm{mM} \mathrm{NaCl}, 1 \mathrm{mM}$ EDTA, $1 \mathrm{mM}$ EDTA, 1\% Triton X100, $20 \mathrm{mM}$ sodium orthovanadate, $1 \mathrm{mM}$ sodium fluoride, $1 \mathrm{mM}$ PMSF, and 1\% protein inhibitor cocktail acquired from Sigma-Aldrich (Madrid, Spain). Homogenates were centrifuged at $10,000 \times \mathrm{g}$ for $2 \mathrm{~min}$ at $4^{\circ} \mathrm{C}$ and the supernatant was stored at $-80^{\circ} \mathrm{C}$ until analysis.

2.4. Western Blot Analysis. Immunoblotting with left ventricular homogenates was used to assess the total (T-) and phosphorylated (p-) levels of NFATc4, AKT, ERK, NF-kB, JNK, CREB, and SERCA. Forty milligrams of left ventricular protein was separated by SDS-PAGE electrophoresis on $12 \%$ gel using the Mini-Protean Tetra system (Bio-Rad, Madrid, Spain) at $100 \mathrm{~V}$ and room temperature. Separated proteins were transferred onto an Immunoblot PVDF membrane (Bio-Rad, Madrid, Spain) for $1 \mathrm{~h}$ at $120 \mathrm{~V}$ and $4^{\circ} \mathrm{C}$. The efficiency of protein transfer was verified using reversible protein and a stain kit (MemCode, Thermo Scientific, Madrid, Spain). Primary antibodies against total (T) and phosphorylated (p) NFATc4 (Santa Cruz Biotechnology, Germany; dilution factor $[\mathrm{DF}]=1: 200)$ and $\mathrm{p}$-AKT $(\mathrm{DF}=1: 1000)$, $\mathrm{p}$-ERK $(\mathrm{DF}=1: 2000), \mathrm{p}-\mathrm{NF}-\kappa \mathrm{B}(\mathrm{DF}=1: 1000), \mathrm{p}-\mathrm{SNP} / \mathrm{JNK}, \mathrm{p}-$ CREB $(\mathrm{DF}=1: 1000)$, and SERCA $(\mathrm{DF}=1: 1000)$ were acquired from Cell Signaling Technology (MA, USA). Primary antibodies were incubated at $4^{\circ} \mathrm{C}$ overnight. After washing with $1 \times$ PBS, the PVDF membrane was incubated with IgG-peroxidase-conjugated secondary anti-rabbit (DF $=1: 2000)$ and anti-mouse (DF $=1: 2000)$ antibodies (Cell Signaling Technology, USA) overnight at room temperature. Blots were washed again and incubated with the SuperSignal West Pico Chemiluminescent substrate kit (Thermo Scientific, Madrid, Spain). Protein expression bands were acquired with a gel documentation and analysis system (Alliance, Uvitec, Cambridge, UK). Density of the bands on the film was analyzed using the free ImageJ NIH software application. GAPDH antibody (Millipore, Madrid, Spain; DF = 1:2000) was used to normalize expression values to correct for protein loading.

\subsection{Biomarkers of Oxidative Stress}

2.5.1. Total Protein Carbonyls. Total protein carbonyls in homogenized left ventricular tissue were quantified using a 
simplified 2,4-dinitrophenylhydrazine (DNPH) spectrophotometric assay [16], which was adapted to nanovolume. Briefly, $2 \mu \mathrm{L}$ of homogenate was incubated with an equal volume of $10 \mathrm{mM} \mathrm{DNPH}$ (in $2.5 \mathrm{~N} \mathrm{HCl}$ ) at room temperature for $10 \mathrm{~min}$, and $1 \mu \mathrm{L}$ sodium hydroxide $(6 \mathrm{~N})$ was then added to the mixture, which was incubated again for $10 \mathrm{~min}$ at room temperature. Absorbance at $450 \mathrm{~nm}$ was read immediately in a Nanodrop 2000 spectrophotometer (Thermo Scientific, NC, USA). Protein carbonyl levels were calculated using the extinction coefficient of DNPH at $450 \mathrm{~nm}(\varepsilon$ $=22308 \mathrm{M}^{-1} \mathrm{~cm}^{-1}$ ) and optical path length of $1 \mathrm{~mm}$ and expressed as nmol of carbonyls/mg protein. The time of incubation of samples after addition of sodium hydroxide was synchronized so that it was $10 \mathrm{~min}$ for all samples, because DNPH is unstable under alkaline conditions. The total protein of homogenates was assessed using Bradford reagent according to the manufacturer's recommendations (Bio-Rad, USA).

2.5.2. Total Thiols. Left ventricular total thiol levels were assessed using the 5, $5^{\prime}$-dithiobis (2-nitrobenzoic acid) assay [17] adapted to nanovolume. Absorbance was measured at $412 \mathrm{~nm}$ in a Nanodrop 2000 spectrophotometer (Thermo Scientific, NC, USA), and total thiol content was expressed as millimoles per liter of reduced glutathione per milligram of protein.

2.5.3. Total Antioxidant Capacity (TAC). Total antioxidant capacity of left ventricular homogenates was assessed using the CUPRAC-BCS assay [18] adapted to nanovolume. Absorbance at $490 \mathrm{~nm}$ was read in a Nanodrop $2000 \mathrm{spec}-$ trophotometer (Thermo Scientific, NC, USA). Total antioxidant capacity values were obtained from the standard curve of the antioxidant trolox $(0-2 \mathrm{~mol} / \mathrm{L})$ and expressed as $\mu \mathrm{mol} / \mathrm{L}$ Trolox.

2.5.4. Catalase Activity. Catalase activity was assessed using the Amplex Red assay (Invitrogen, Spain) adapted to a 384well plate. Absorbance at $560 \mathrm{~nm}$ was read after $30 \mathrm{~min}$ of reaction at room temperature in a multimode microplate reader (Synergy HT, Bio-Tek, VT, USA). Catalase activity was expressed as $\mathrm{U} / \mathrm{mg}$ protein, calculated from the CAT standard curve $(0-2 \mathrm{U} / \mathrm{mL})$.

2.5.5. Protein Content. Protein content was assessed using the Coomassie blue-based microtiter plate assay according to the manufacturer's recommendations (Bio-Rad, Madrid, Spain). Absorbance was measured at $595 \mathrm{~nm}$ in a Synergy HT Multimode Microplate Reader (Bio-tek), and bovine serum albumin was used as the standard.

2.6. Statistical Analysis. The results were expressed as the mean \pm SEM. The parameters were compared using repeated measures analysis of variance (physiological parameters) or single-factor (rat) analysis of variance (western blot analysis and oxidative stress parameters). A post hoc Bonferroni correction was applied. Statistical significance was set at $P \leq$ 0.05 . The analysis was performed using IBM SPSS Statistics

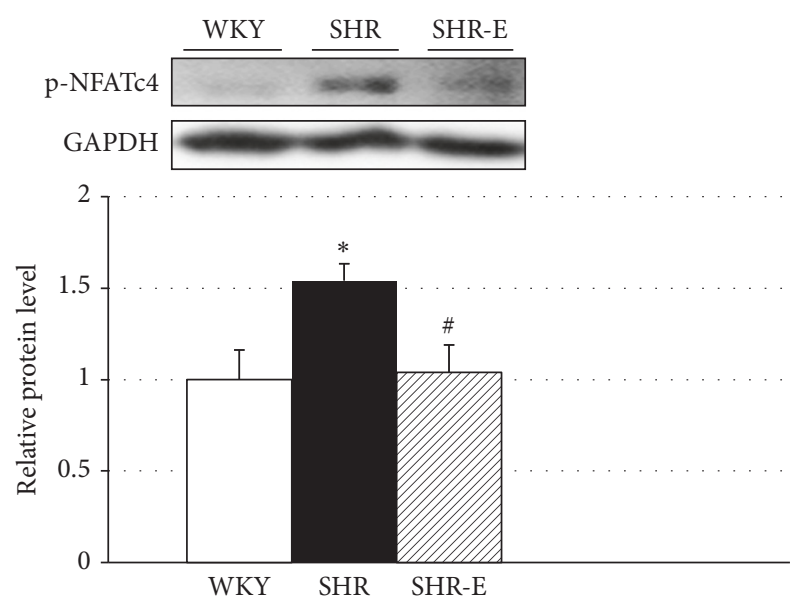

FIgURE 1: Expression levels of p-NFATc4 in the left ventricle. Experiments were performed in homogenates of left ventricular tissue from Wistar-Kyoto rats (WKY), spontaneously hypertensive rats (SHR), and esmolol-treated SHRs (SHR-E). Values are shown as mean \pm SEM; $n=6 .{ }^{*} P<0.05$ versus WKY and ${ }^{\#} P<0.05$ versus untreated SHR

for Windows, version 20.0 (IBM Corp, Armonk, New York, USA) and Prism GraphPad 6.0 (GraphPad Software, California, USA).

\section{Results}

3.1. Blood Pressure and Heart Rate Measurements. Values of physiological parameters are shown in Table 1. Systolic arterial pressure was significantly higher in SHR than in WKY controls $(P<0.01)$. Short-term administration of esmolol for 48 hours significantly reduced systolic arterial pressure in SHR-E during the $48 \mathrm{~h}$ of treatment, and these values were comparable to those of the WKY. No statistically significant differences in heart rate were found between WKY and SHR, and heart rate was reduced in SHR-E during the $48 \mathrm{~h}$ of treatment with respect to WKY $(P<0.001)$ and SHR $(P<$ 0.001).

3.2. Short-Term Administration of Esmolol Decreases NFATc4 Phosphorylation in the Left Ventricular Myocardial Tissue of Adult Spontaneously Hypertensive Rats. In the whole tissue left ventricular homogenates, p-NFATc4 levels were markedly higher in SHR than in WKY controls, and short-term treatment of SHR with esmolol significantly reduced p-NFATc4 levels (Figure 1, $P<0.05$ ). Serca2a and $\mathrm{p}$-Creb1 expression in the left ventricle of untreated and treated rats were similar (Figures 2(a) and 2(b)). This finding may suggest a decrease in the cardiac hypertrophy signaling in adult SHR.

3.3. Short-Term Administration of Esmolol Decreases Akt and NF-kB Phosphorylation in the Left Ventricle of Adult SHR. pAkt and p-NF-kB expression in the left ventricular tissue was lower in SHR-E than in SHR and treatment with esmolol reduced this expression to the protein levels of WKY (Figures 3(a) and 3(b)). p-Akt levels were higher in SHR versus WKY 
TABLE 1: Systolic blood pressure and heart rate in WKY, SHR, and SHR-E.

\begin{tabular}{|c|c|c|c|}
\hline Groups & Time treatment (hours) & $\mathrm{SBP}(\mathrm{mmHg})$ & HR (bpm) \\
\hline \multirow{5}{*}{ WKY } & 0 & $126 \pm 21$ & $310 \pm 20$ \\
\hline & 12 & $135 \pm 18$ & $317 \pm 15$ \\
\hline & 24 & $119 \pm 11$ & $324 \pm 21$ \\
\hline & 36 & $121 \pm 22$ & $319 \pm 19$ \\
\hline & 48 & $127 \pm 23$ & $320 \pm 12$ \\
\hline \multirow{5}{*}{ SHR } & 0 & $190 \pm 20^{* *}$ & $322 \pm 15$ \\
\hline & 12 & $217 \pm 25^{* *}$ & $311 \pm 11$ \\
\hline & 24 & $198 \pm 21^{* *}$ & $324 \pm 18$ \\
\hline & 36 & $223 \pm 12^{* * *}$ & $312 \pm 20$ \\
\hline & 48 & $205 \pm 23^{* *}$ & $313 \pm 16$ \\
\hline \multirow{5}{*}{ SHR-E } & 0 & $199 \pm 31^{* *}$ & $320 \pm 13$ \\
\hline & 12 & $137 \pm 15^{\# \#}$ & 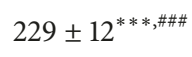 \\
\hline & 24 & $139 \pm 28^{\# \#}$ & $231 \pm 10^{* * *, \# \# \#}$ \\
\hline & 36 & $145 \pm 11^{\# \#}$ & $219 \pm 18^{* * *, \# \# \#}$ \\
\hline & 48 & $143 \pm 10^{\# \#}$ & $220 \pm 13^{* * *, \# \# \#}$ \\
\hline
\end{tabular}

WKY: Wistar-Kyoto rats; SHR: spontaneously hypertensive rats; SHR-E: esmolol-treated SHR rats; SBP: systolic blood pressure; HR: heart rate. Values are shown as mean \pm SEM. $n=6 .{ }^{* *} P<0.01$ versus WKY and ${ }^{* * *} P<0.001$ versus WKY; ${ }^{\# \#} P<0.01$ versus untreated SHR and ${ }^{\# \# \#} P<0.001$ versus untreated SHR.

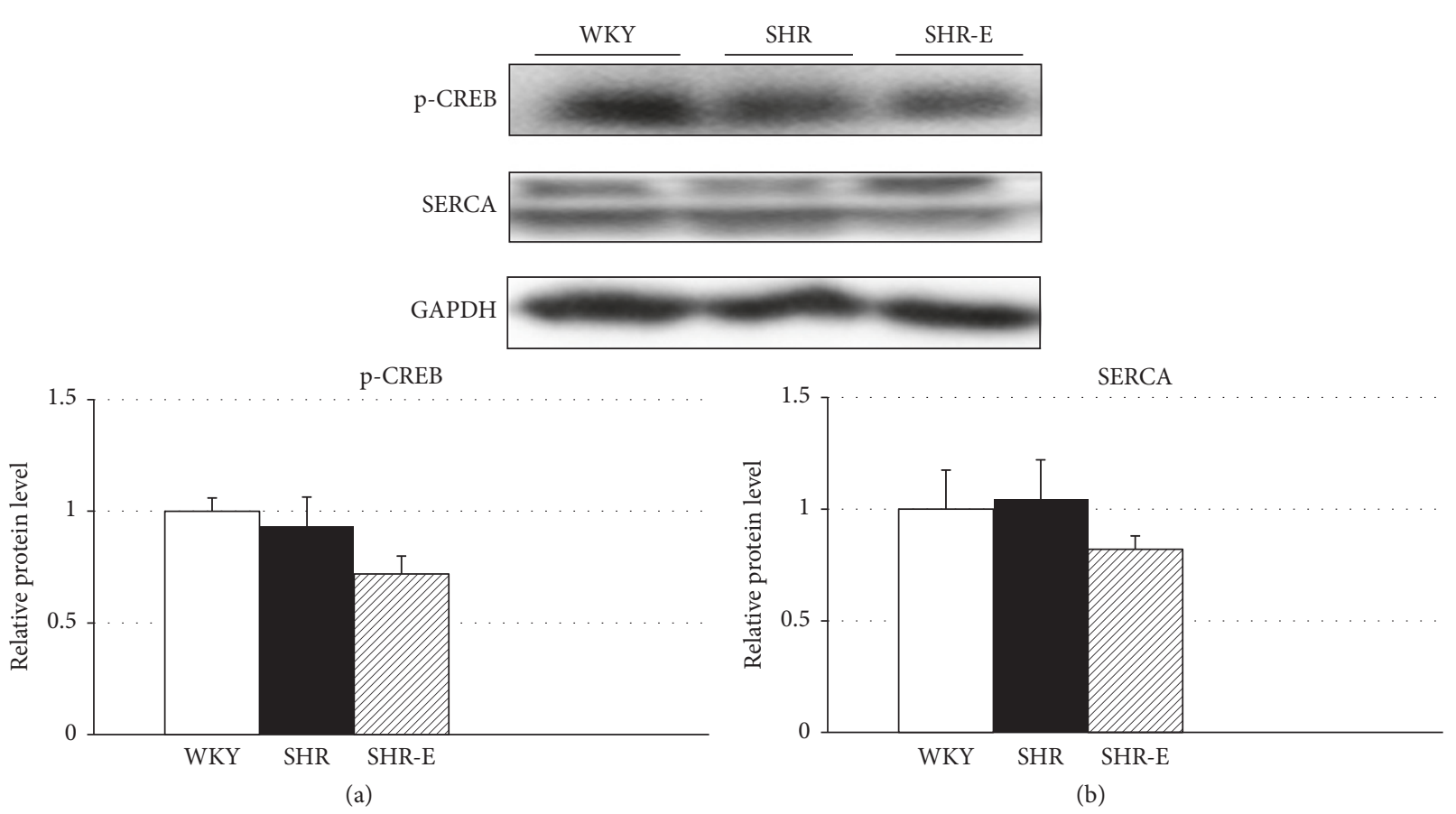

FIGURE 2: Expression levels of p-Crebl and Serca2a in the left ventricle. Experiments were performed in homogenates of left ventricular tissue from Wistar-Kyoto rats (WKY), spontaneously hypertensive rats (SHR), and esmolol-treated SHRs (SHR-E). Values are shown as mean \pm SEM; $n=6$.

(Figure 3(a), $P<0.05)$. p-NF-kB levels were higher in SHR versus WKY (Figure 3(b), $P<0.05$ ). No differences in p-Sapk/Jnk expression were observed when comparing the three experimental groups (Figure 3(d)). There was a small trend in p-Erk1/2 increase in the SHR group but it was not statistically significant (Figure 3(c)).
3.4. Short-Term Administration of Esmolol Reduces Oxidative Stress and Increases Catalase Activity in the Left Ventricular Tissue. Short-term treatment with esmolol restored catalase activity to the levels in WKY control heart tissue. Compared to WKY rats and SHR-E, catalase activity was decreased in SHR animals (Figure 4(a), $P<0.01$ ). Protein carbonyl levels 


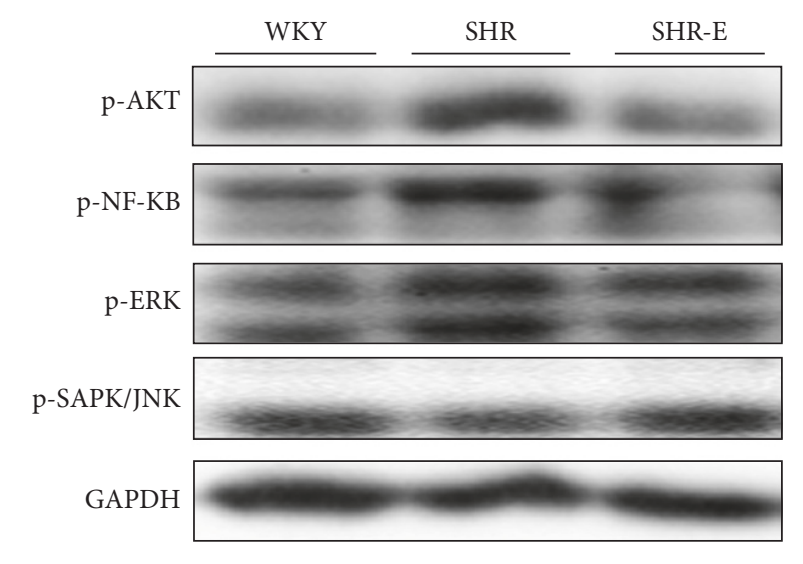

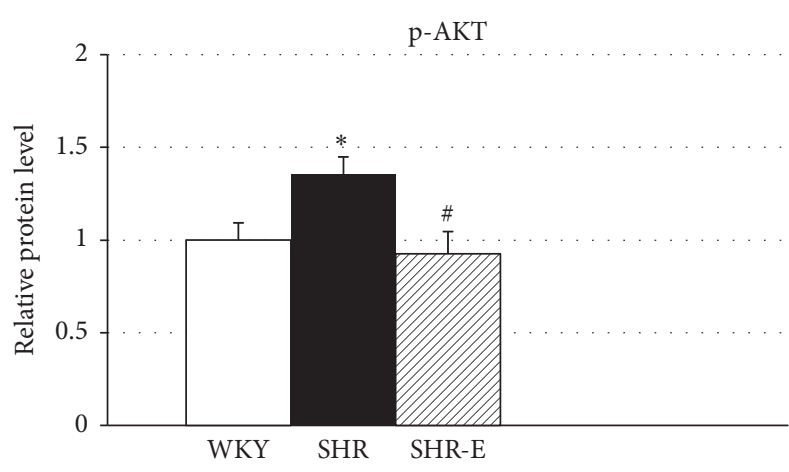

(a)

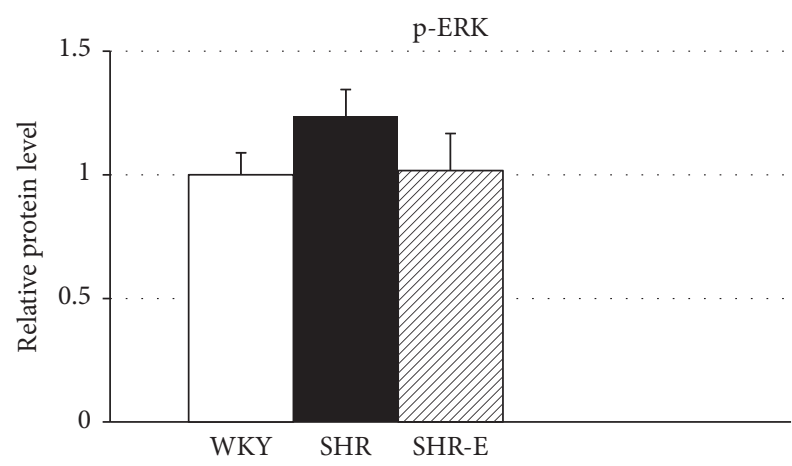

(c)

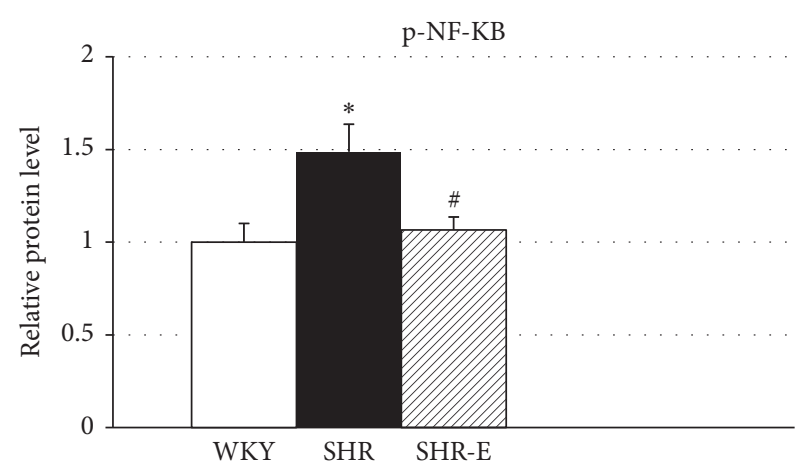

(b)

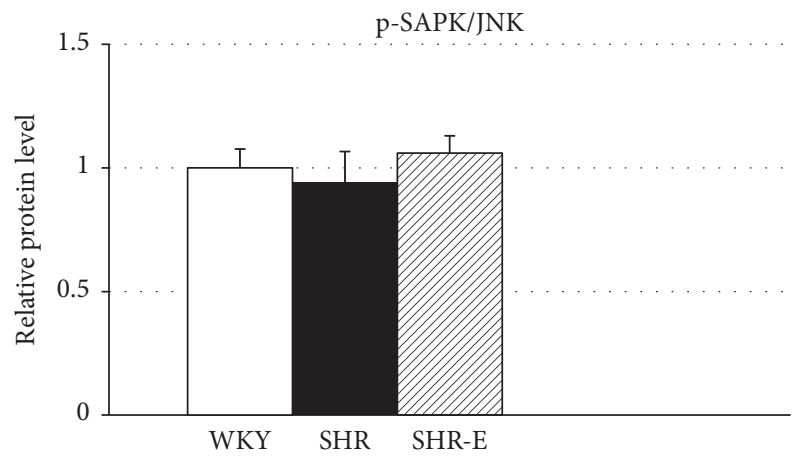

(d)

Figure 3: Expression levels of p-Akt (a), p-NF- $\kappa$ B (b), p-Erk1/2 (c), and p-Sapk/Jnk (d) in left ventricular tissue. Experiments were performed using left ventricular homogenates from Wistar-Kyoto rats (WKY), spontaneously hypertensive rats (SHR), and esmolol-treated SHRs (SHRE). Values are shown as mean $\pm \mathrm{SEM} ; n=6{ }^{*} P<0.05$ versus $\mathrm{WKY}$ and ${ }^{\#} P<0.05$ versus untreated SHR.

in the left ventricular tissue of SHR-E were lower than in SHR and WKY rat hearts (Figure $4(\mathrm{~b}), P<0.05$ ), and comparable in SHR and WKY (Figure 4(b)). No differences were observed in total thiols and total antioxidant capacity in the three experimental groups (Figures 4(c) and 4(d)).

\section{Discussion}

We previously reported that short-term treatment with esmolol produces early regression of left ventricular hypertrophy (LVH) in SHR [5]. Esmolol led to early reduction of left ventricular mass, early changes in the cross-sectional area of left ventricular cardiomyocytes, and a marked decrease in glucose metabolism in the hypertrophied ventricle [5]. We recently showed that esmolol reduces ADMA concentrations and increases DDAH concentrations in the left ventricle in SHR $[10,11]$. These findings would explain, in part, the regression of LVH, although several mechanisms could be involved in regression of $\mathrm{LVH}$ with esmolol. In the present study we show that short-term administration of esmolol reversed LVH in adult SHR at least in part by downregulation of Akt/NF- $\kappa$ B and NFATc4 protein phosphorylation and reduced oxidative stress. It also increases catalase activity in the left ventricular tissue.

Induction of the calcineurin-NFAT pathway is a common hallmark of pathological hypertrophy [19-21]. Calcineurin 


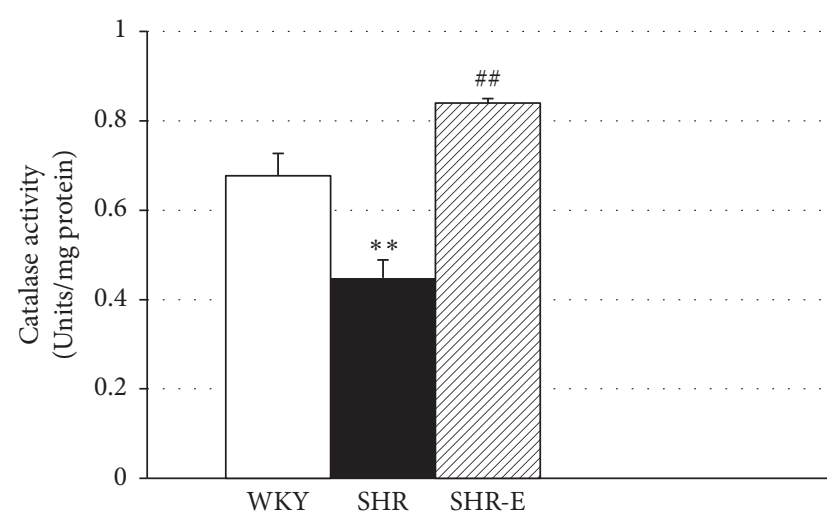

(a)

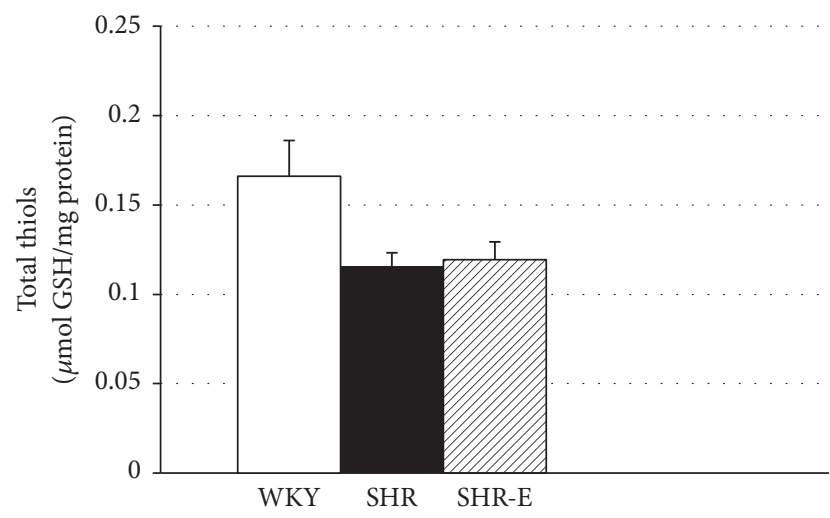

(c)

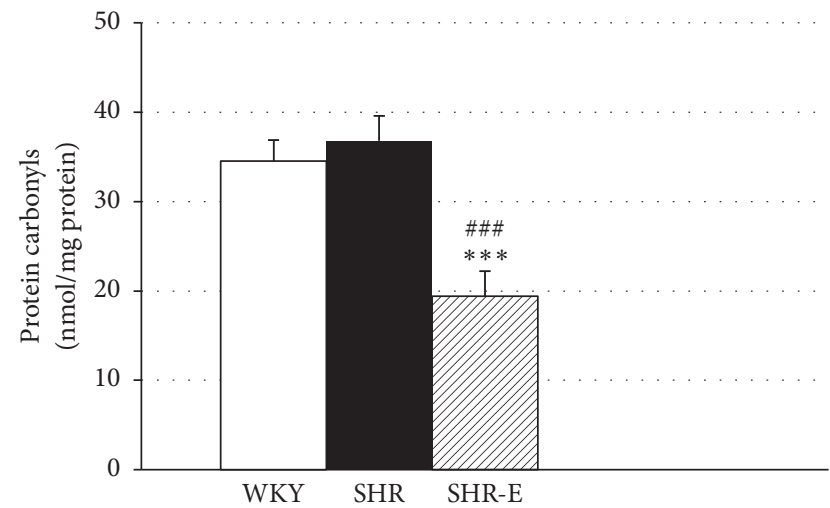

(b)

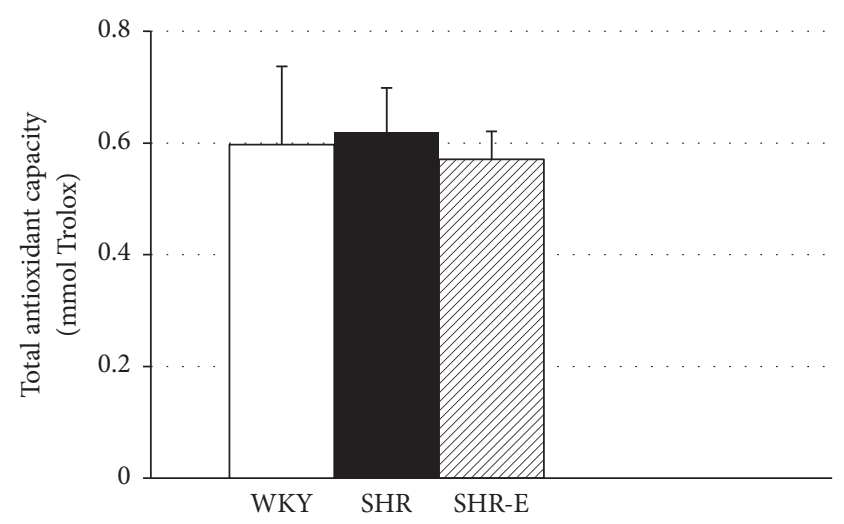

(d)

FIGURE 4: Left ventricular oxidative stress biomarkers: catalase activity (a), protein carbonyls (b), total thiols (c), and total antioxidant capacity (d). Experiments were performed in left ventricular homogenates from Wistar-Kyoto rats (WKY), spontaneously hypertensive rats (SHR), and esmolol-treated SHRs (SHR-E). Values are shown as mean \pm SEM; $n=6$. ${ }^{* *} P<0.01$ versus WKY, ${ }^{* * *} P<0.001$ versus WKY and ${ }^{\# \#} P<0.01$ versus untreated SHR, and ${ }^{\# \# \#} P<0.001$ versus untreated SHR.

is a calcium/calmodulin-activated serine/threonine phosphatase that once stimulated de-phosphorylates and thereby activates the NFAT transcription factor. Dephosphorylated NFAT translocate from the cytoplasm to the nucleus which leads to the transcription of cardiac hypertrophy-associated genes [19-21]. We found that p-NFATc4 was markedly higher in left ventricular heart tissue from SHR compared to WKY. NFATc4, one of five NFAT family members, was shown to be capable of promoting cardiac hypertrophy in vivo. NFATc4 functions as a calcineurin effector by trafficking between the cytosol and the nucleus to regulate the cardiac hypertrophic response [22]. Although p-NFATc4 levels in cytoplasm are decreased as a consequence of nuclear translocation in cardiomyocytes, studies on protein expression in left ventricular whole heart tissue in hypertrophic animal models are scarce. NFATc4 has been described as a new mechanism that contributes to the induction of pathological cardiac hypertrophy [22] Interestingly, short-term treatment of SHR with esmolol significantly reduced p-NFATc4 levels.

Esmolol is an unique cardioselective $\beta 1$-receptor blocking agent with a rapid onset and short duration of action that has been tested in a variety of patients, including those with unstable angina, myocardial ischemia, supraventricular arrhythmias, and peri- and postoperative tachycardia and hypertension [6-8]. In surgical and critical care setting where clinical conditions are rapidly changing, the pharmacokinetic profile of esmolol (it has a very short half-life and a short duration of action) allows the drug to provide rapid pharmacologic control and minimizes the potential for serious adverse effects. A meta-analysis of 67 randomized clinical trials shows that esmolol effectively decreased both heart rate and arterial blood pressure [8]. Intravenous esmolol has been shown to reduce the incidence of myocardial ischemia and arrhythmias in cardiac (a total of 778 patients from 20 randomized trials) and noncardiac surgery (a total of 1765 patients from 32 randomized trials) without increasing the episodes of hypotension and bradycardia $[23,24]$. One proposed benefit of $\beta$-receptor blockade is a myocyte-autonomous reduction in hypertrophy and cell death [21]. Activating $\beta$-adrenergic receptors for extended periods of time (hypertension, heart failure, or volume overload due to valve dysfunction) leads to hypertrophy and $\beta$-receptor desensitization [21, 25]. Furthermore, the agerelated reduction in cardiac $\beta$-adrenergic receptor sensitivity 
and density has been reported in the myocardium [6]. In the present study, $\beta$-adrenergic receptor desensitization was demonstrated by the similar expression of Serca2a and $\mathrm{p}$ Crebl observed in the left ventricle of untreated and treated aged SHR [21]. A previous study has also shown that SHR and WKY rats showed similar Serca2a levels [26, 27]. Therefore, short-term treatment with esmolol can regulate cardiac hypertrophy in adult SHR through regulation of molecular pathways other than $\beta$-adrenergic blockade, as suggested elsewhere [21].

Calcineurin-NFAT and PI3K/Akt signaling pathways are interdependent and together orchestrate the cardiac hypertrophic response [21]. The $\mathrm{NF} \kappa \mathrm{B}$ signaling cascade interacts with PI3K/Akt signaling pathways [28]. We found that levels of p-Akt in the left ventricular tissue were lower in SHR-E than in SHR. Upregulation of Akt can contribute to adaptive cardiac hypertrophy; however, persistent Akt activation over longer period of time can lead to unsustainable cardiac hypertrophy signaling that may develop into decompensation leading to heart failure [29]. In contrast to our findings, levels of p-Akt were reported to be lower for SHR than for normotensive WKY [30, 31]. Similarly, esmolol reduced pNF- $\kappa \mathrm{B}$ expression to the protein level of WKY. NF- $\kappa \mathrm{B}$ has also been postulated to play a role in cardiac hypertrophy $[20,32]$.

The Akt pathway is actively involved in the regulation of NF- $\kappa \mathrm{B}$ [33]. Akt can activate NF- $\kappa \mathrm{B}$ by inducing phosphorylation [28], and $\mathrm{NF}-\kappa \mathrm{B}$ inhibition attenuates cardiac hypertrophy [34]. A recent study demonstrated that NFATc4 and NF- $\kappa \mathrm{B}$ could interact and assemble a transcriptional complex that effectively coordinates cardiac hypertrophy and pathological remodeling [35]. Thus, it was recently observed that the nuclear translocation of NFATc4 and the transcriptional activity of NF- $\kappa \mathrm{B}$ were significantly increased by phenylephrine, which was significantly inhibited by pretreatment with the known antioxidant alpha-lipoic acid [36].

In a previous study, our group has demonstrated that, compared with SHR, plasma superoxide anion scavenging activity (including superoxide dismutase) was more pronounced in SHR-E and this was associated with an improvement in nitric oxide availability measured as nitrites/nitrates [37]. In the present study, short-term administration of esmolol restored catalase activity to the level detected in WKY controls. Catalase activity was less pronounced in SHR than in WKY and SHR-E, thus reflecting the possibility that esmolol also increases superoxide dismutase activity in the left ventricular tissue because an increase in hydrogen peroxide may induce catalase activity. Indeed, catalase activity in blood is a good indicator of the redox status of the heart [38]. NADPH oxidase (Nox2) was found to be essential for angiotensin II-induced cardiac hypertrophy [39], which is involved in the enhanced activation of MAPKs and the NF$\kappa \mathrm{B}$ pathway [12]. Protein carbonyl levels were also lower in the left ventricular tissue of SHR-E, although they remained unchanged in untreated SHR and WKY. Finally, similar to findings from our previous study based on plasma from SHR-E [37], no differences in total antioxidant capacity or total thiols were observed between the three experimental groups.
Left ventricular hypertrophy $(\mathrm{LVH})$ is probably the most visible manifestation of hypertensive organ damage [1]. Several clinical and experimental animal studies have shown that cardiac hypertrophy is reversed by various antihypertensive drugs (angiotensin-converting enzyme inhibitors, angiotensin receptor blockers, $\beta$-adrenergic blockers, calcium channel blockers, and diuretics), although they were based on long-term therapy. On the other hand, a better understanding of the cellular and molecular mechanisms underlying cardiac hypertrophy is critical when attempting to identify new therapeutic targets to inhibit LVH. We report the results of the first study to associate regression of $\mathrm{LVH}$ after a markedly short administration, 48 hours, of a $\beta$-blocker esmolol [5]. However, it is necessary to study in more detail the underlying molecular mechanisms of positive effect of esmolol on inhibition of $\mathrm{LVH}$ before testing this treatment modality/regimen in humans. If these effects of short esmolol administration could be confirmed in humans, esmolol could be an option for the treatment of LVH in critical care units.

In conclusion, our findings show that short-term administration of esmolol reverses LVH in adult SHR through inhibition of Akt/NF- $\kappa$ B and NFATc4 phosphorylation, reduction of oxidative stress, and increased catalase activity in the left ventricular cardiac tissue.

\section{Conflicts of Interest}

The authors declare that they have no conflicts of interest.

\section{Acknowledgments}

This study was supported by Spanish Health Ministry (Fondo de Investigaciones Sanitarias) under Contracts FIS 13/01261 and FIS 16/02069 and Fondos Feder.

\section{References}

[1] G. Y. Lip, "Regression of left ventricular hypertrophy and improved prognosis: some hope now ... or hype?" Circulation, vol. 104, pp. 1582-1584, 2001.

[2] S. M. Artham, C. J. Lavie, R. V. Milani, D. A. Patel, A. Verma, and H. O. Ventura, "Clinical Impact of Left Ventricular Hypertrophy and Implications for Regression," Progress in Cardiovascular Diseases, vol. 52, no. 2, pp. 153-167, 2009.

[3] R. H. Fagard, H. Celis, L. Thijs, and S. Wouters, "Regression of left ventricular mass by antihypertensive treatment: A metaanalysis of randomized comparative studies," Hypertension, vol. 54, no. 5, pp. 1084-1091, 2009.

[4] C. Ferreira Filho, LC. Abreu, VE. Valenti et al., "Antihypertensive drugs have different effects on ventricular hypertrophy regression," Clinics, vol. 65, pp. 723-728, 2010.

[5] B. Quintana-Villamandos, M. J. Delgado-Martos, J. J. SanchezHernandez et al., "Early regression of left ventricular hypertrophy after treatment with esmolol in an experimental rat model of primary hypertension," Hypertens Res, vol. 36, pp. 408-413, 2013.

[6] D. B. Wiest and J. S. Haney, "Clinical pharmacokinetics and therapeutic efficacy of esmolol," Clinical Pharmacokinetics, vol. 51, no. 6, pp. 347-356, 2012. 
[7] S. K. Yu, G. Tait, K. Karkouti, D. Wijeysundera, S. McCluskey, and W. S. Beattie, "The safety of perioperative esmolol: a systematic review and meta-analysis of randomized controlled trials," Anesth Analg, vol. 112, pp. 267-281, 2011.

[8] K. P. Garnock-Jones, "Esmolol: A Review of its Use in the Short-Term Treatment of Tachyarrhythmias and the ShortTerm Control of Tachycardia and Hypertension," Drugs, vol. 72, no. 1, pp. 109-132, 2012.

[9] Y. Bai and R. Hui, "Dimethylarginine dimethylaminohydrolase (DDAH) - a critical regulator of hypertensive left ventricular hypertrophy?" Medical Hypotheses, vol. 70, no. 5, pp. 962-966, 2008.

[10] B. Quintana-Villamandos, A. Arnalich-Montiel, S. Arribas et al., "Early regression of coronary artery remodeling with esmolol and DDAH/ADMA pathway in hypertensive rats," Hypertension Research, vol. 39, no. 10, pp. 692-700, 2016.

[11] B. Quintana-Villamandos and E. Delgado-Baeza, "Does the ADMA/DDAH/NO pathway modulate early regression of left ventricular hypertrophy with esmolol?" Medical Hypotheses, vol. 87, pp. 44-47, 2016.

[12] C. M. Sag, C. X. C. Santos, and A. M. Shah, "Redox regulation of cardiac hypertrophy," Journal of Molecular and Cellular Cardiology, vol. 73, pp. 103-111, 2014.

[13] A. M. Shah and D. L. Mann, "In search of new therapeutic targets and strategies for heart failure: Recent advances in basic science," The Lancet, vol. 378, no. 9792, pp. 704-712, 2011.

[14] J. Heineke and J. D. Molkentin, "Regulation of cardiac hypertrophy by intracellular signalling pathways," Nature Reviews Molecular Cell Biology, vol. 7, no. 8, pp. 589-600, 2006.

[15] F. Simko and O. Pechanova, "Remodelling of the heart and vessels in experimental hypertension: advances in protection," Journal of Hypertension, vol. 28, supplement 1, pp. S1-S6, 2010.

[16] C. S. Mesquita, R. Oliveira, F. Bento, D. Geraldo, J. V. Rodrigues, and J. C. Marcos, "Simplified 2,4-dinitrophenylhydrazine spectrophotometric assay for quantification of carbonyls in oxidized proteins," Analytical Biochemistry, vol. 458, pp. 69-71, 2014.

[17] L. Condezo-Hoyos, M. Rubio, S. M. Arribas et al., "A plasma oxidative stress global index in early stages of chronic venous insufficiency," Journal of Vascular Surgery, vol. 57, no. 1, pp. 205213, 2013.

[18] C. Campos, R. Guzmán, E. López-Fernández, and Á. Casado, "Evaluation of the copper(II) reduction assay using bathocuproinedisulfonic acid disodium salt for the total antioxidant capacity assessment: the CUPRAC-BCS assay," Analytical Biochemistry, vol. 392, no. 1, pp. 37-44, 2009.

[19] W. E. Stansfield, M. Ranek, A. Pendse et al., The Pathophysiology of Cardiac Hypertrophy and Heart Failure. Cellular and Molecular Pathobiology of Cardiovascular Disease, Chapter 4, Academic Press, San Diego, calif, USA, 2014.

[20] B. C. Bernardo, K. L. Weeks, L. Pretorius, and J. R. McMullen, "Molecular distinction between physiological and pathological cardiac hypertrophy: experimental findings and therapeutic strategies," Pharmacology \& Therapeutics, vol. 128, no. 1, pp. 191227, 2010.

[21] J. H. van Berlo, M. Maillet, and J. D. Molkentin, "Signaling effectors underlying pathologic growth and remodeling of the heart," The Journal of Clinical Investigation, vol. 123, no. 1, pp. 37-45, 2013.

[22] C. Li, J. Li, X. Cai et al., "Protein kinase D3 is a pivotal activator of pathological cardiac hypertrophy by selectively increasing the expression of hypertrophic transcription factors," The Journal of Biological Chemistry, vol. 286, no. 47, pp. 40782-40791, 2011.
[23] A. Zangrillo, S. Turi, G. Crescenzi et al., "Esmolol Reduces Perioperative Ischemia in Cardiac Surgery: A Meta-analysis of Randomized Controlled Studies," Journal of Cardiothoracic and Vascular Anesthesia, vol. 23, no. 5, pp. 625-632, 2009.

[24] G. Landoni, S. Turi, G. Biondi-Zoccai et al., "Esmolol Reduces Perioperative Ischemia in Noncardiac Surgery: A Meta-analysis of Randomized Controlled Studies," Journal of Cardiothoracic and Vascular Anesthesia, vol. 24, no. 2, pp. 219-229, 2010.

[25] B. Strulovici, R. A. Cerione, B. F. Kilpatrick, M. G. Caron, and R. J. Lefkowitz, "Direct demonstration of impaired functionality of a purified desensitized $\beta$-adrenergic receptor in a reconstituted system," Science, vol. 225, no. 4664, pp. 837-840, 1984.

[26] S. M. MacDonnell, H. Kubo, D. L. Crabbe et al., "Improved myocardial $\beta$-adrenergic responsiveness and signaling with exercise training in hypertension," Circulation, vol. 111, no. 25, pp. 3420-3428, 2005.

[27] P. Boknik, I. Heinroth-Hoffmann, U. Kirchhefer et al., "Enhanced protein phosphorylation in hypertensive hypertrophy," Cardiovascular Research, vol. 51, pp. 717-728, 2001.

[28] D. Bai, L. Ueno, and P. K. Vogt, "Akt-mediated regulation of $\mathrm{NF} \kappa \mathrm{B}$ and the essentialness of $\mathrm{NF} \kappa \mathrm{B}$ for the oncogenicity of PI3K and Akt," International Journal of Cancer, vol. 125, no. 12, pp. 2863-2870, 2009.

[29] P. Abeyrathna and Y. Su, "The critical role of Akt in cardiovascular function," Vascular Pharmacology, vol. 74, pp. 38-48, 2015.

[30] W.-W. Kuo, C.-Y. Chu, C.-H. Wu et al., "Impaired IGF-I signalling of hypertrophic hearts in the developmental phase of hypertension in genetically hypertensive rats," Cell Biochemistry \& Function, vol. 23, no. 5, pp. 325-331, 2005.

[31] W. B. Zhang, Q. J. Du, H. Li et al., "The therapeutic effect of Rosuvastatin on cardiac remodelling from hypertrophy to fibrosis during the end-stage hypertension in rats," Journal of Cellular \& Molecular Medicine, vol. 16, no. 9, pp. 2227-2237, 2012.

[32] N. H. Purcell, G. Tang, C. Yu, F. Mercurio, J. A. DiDonato, and A. Lin, "Activation of NF- $\kappa$ B is required for hypertrophic growth of primary rat neonatal ventricular cardiomyocytes," Proceedings of the National Acadamy of Sciences of the United States of America, vol. 98, no. 12, pp. 6668-6673, 2001.

[33] O. N. Ozes, L. D. Mayo, J. A. Gustin, S. R. Pfeffer, L. M. Pfeffer, and D. B. Donner, "NF- $\kappa$ B activation by tumour necrosis factor requires tie Akt serine-threonine kinase," Nature, vol. 401, no. 6748, pp. 82-85, 1999.

[34] L. Zelarayan, A. Renger, C. Noack et al., "NF- $\kappa$ B activation is required for adaptive cardiac hypertrophy," Cardiovascular Research, vol. 84, no. 3, pp. 416-424, 2009.

[35] Q. Liu, Y. Chen, M. Auger-Messier, and J. D. Molkentin, "Interaction between NFKB and NFAT coordinates cardiac hypertrophy and pathological remodeling," Circulation Research, vol.110, no. 8, pp. 1077-1086, 2012.

[36] J. Zou, X. Gan, H. Zhou et al., "Alpha-lipoic acid attenuates cardiac hypertrophy via inhibition of C/EBP $\beta$ activation," Molecular and Cellular Endocrinology, vol. 399, pp. 321-329, 2015.

[37] A. Arnalich-Montiel, M. C. Gonzalez, E. Delgado-Baeza et al., "Short-term esmolol improves coronary artery remodeling in spontaneously hypertensive rats through increased nitric oxide bioavailability and superoxide dismutase activity," BioMed Research International, vol. 2014, Article ID 531087, 9 pages, 2014.

[38] A. S. Veskoukis, M. G. Nikolaidis, A. Kyparos, and D. Kouretas, "Blood reflects tissue oxidative stress depending on biomarker 
and tissue studied," Free Radical Biology \& Medicine, vol. 47, no. 10, pp. 1371-1374, 2009.

[39] M. Zhang, A. Perino, A. Ghigo, E. Hirsch, and A. M. Shah, "NADPH oxidases in heart failure: poachers or gamekeepers?" Antioxidants \& Redox Signaling, vol. 18, no. 9, pp. 1024-1041, 2013. 


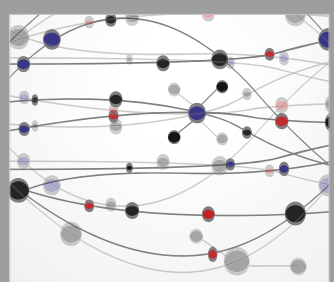

The Scientific World Journal
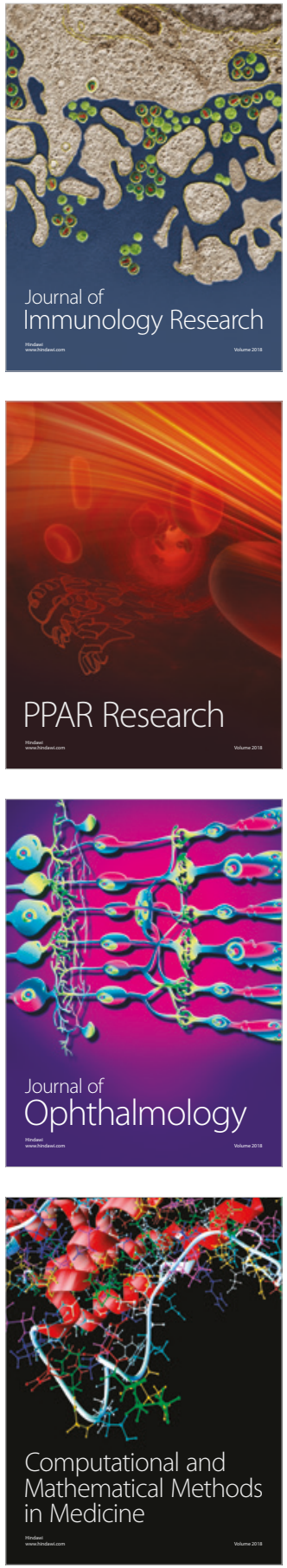

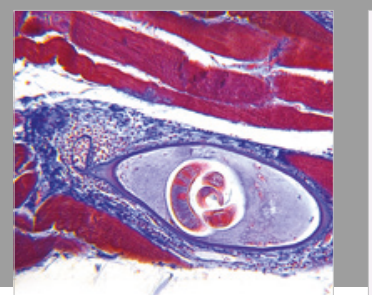

Gastroenterology Research and Practice

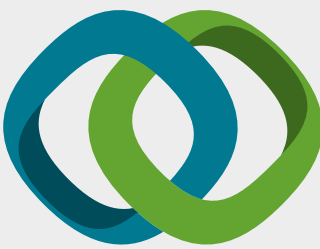

\section{Hindawi}

Submit your manuscripts at

www.hindawi.com
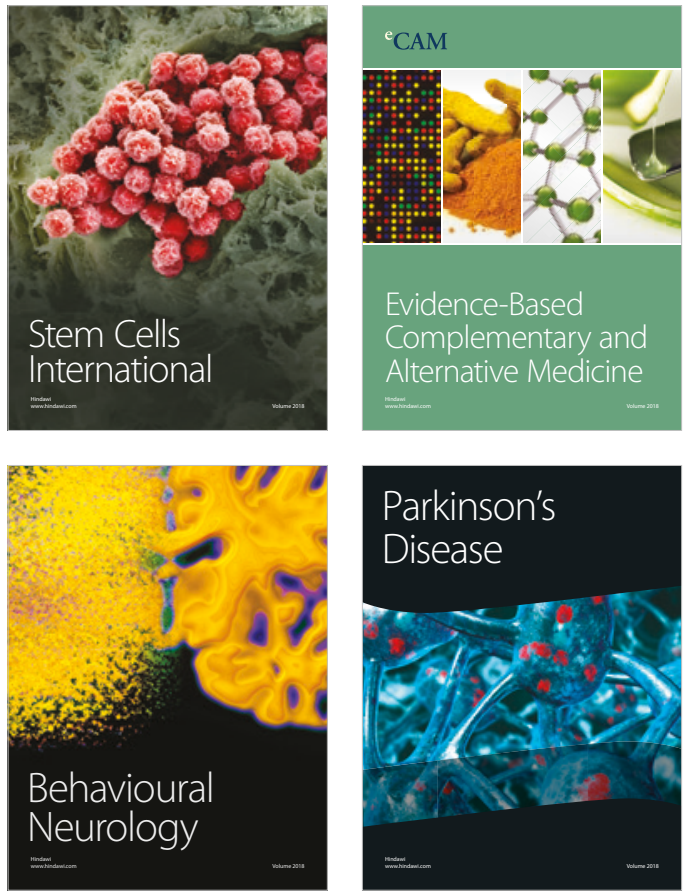

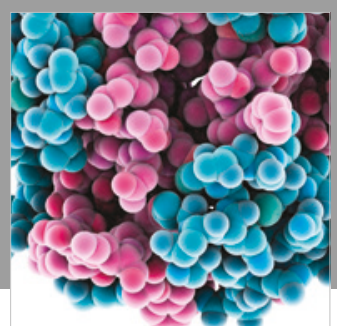

ournal of

Diabetes Research

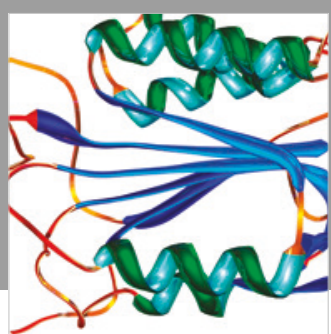

Disease Markers
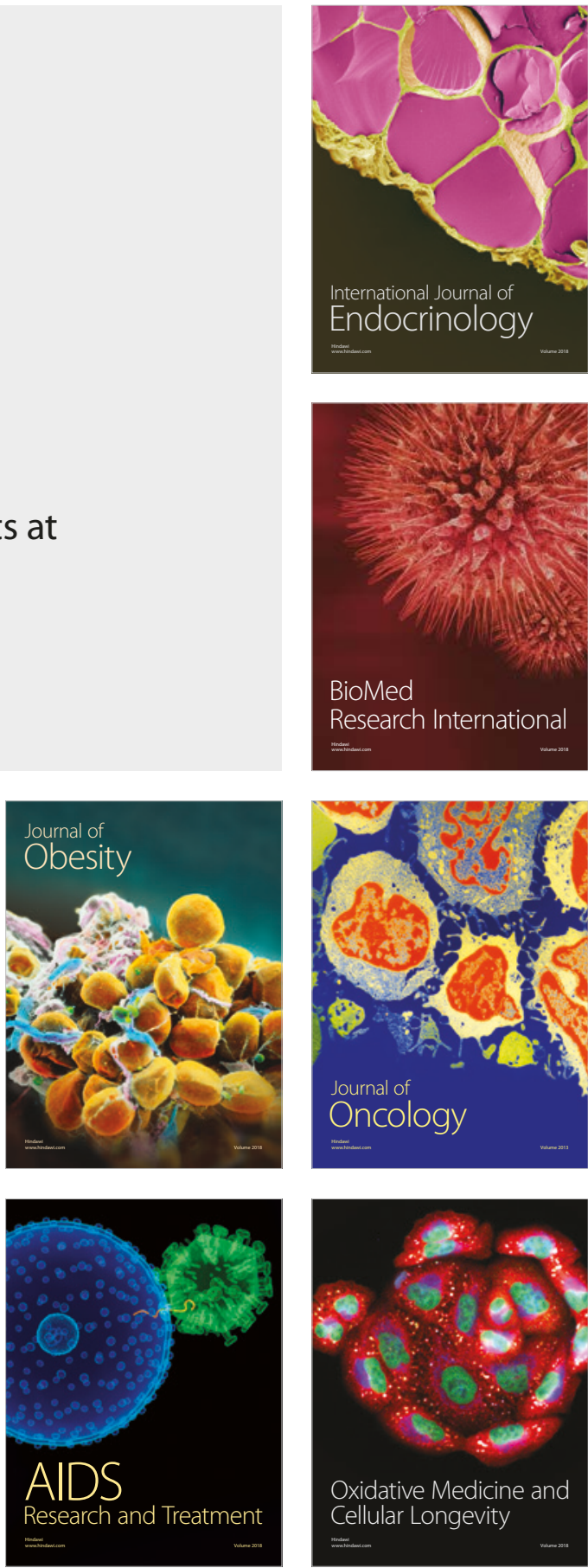